Proc. 15th Int. Conference on Defects Recognition, Imaging and Physics in Semiconductors, Warsaw, Poland 2013

\title{
PeakForce Tapping Technique for Characterization of Thin Organic Passivating Layers
}

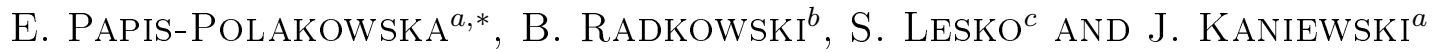 \\ ${ }^{a}$ Institute of Electron Technology, al. Lotników 32/46, 02-668 Warszawa, Poland \\ ${ }^{b}$ Labsoft - Krzysztof Herman, Wantule 12, 02-828 Warszawa, Poland \\ ${ }^{c}$ Bruker Nano Surfaces Division, 112 Robin Hill Road, Santa Barbara, CA 93117, USA
}

\begin{abstract}
The PeakForce Tapping technique was used for study of GaAs and GaSb surfaces treated by hexadecanethiol (HDT) - the sensitive self-assemble compound. The results of both surface morphology control and electrical properties characterization have been presented.
\end{abstract}

DOI: 10.12693/APhysPolA.125.1056

PACS: 07.79.Lh, 64.75.Yz, 81.65.Rv

\section{Introduction}

Atomic force microscopy (AFM) is now widely used in the field of characterization of a variety of materials. In addition to the precise measurement of surface topography with a resolution enabling the observation of atomic steps, current devices of this type offer a number of additional features, including the study of electrical, magnetic and thermal properties. The next generation technology in this field, complementary to already developed AFM technique, which enhance the range of possible applications, including automated mode topographic imaging and mapping modes allows to characterize the surface mechanical properties and electrical conductivity [1].

In particular, the basis of the modes of operation is a technique called PeakForce Tapping, which is using the lever oscillation with a frequency far below resonance, and in such way can carry a surface scan during which each individual contact with the surface of the sample is treated as an independent force spectroscopy experiment. Each contact of the tip with the surface forms an individual force distance curve that is immediately analyzed using the unique software and electronics to enable realization of such measurements. In this way, the most important factor associated with the implementation of this technique of measurement - the force of the contact between the tip and the surface of the sample - is controlled in real-time.

Thanks to these innovations the area of application of atomic force microscopy has been expanded significantly, allowing testing of materials that previously were not suitable for the use of such techniques. This concerns the thin organic monolayers on semiconductors, especially the sensitive self-assemble compounds, like thiols or thioacetamide.

Generally, the characterization of organic monolayers typically involves a combination of multiple techniques, like Fourier transform infrared spectroscopy (FTIR),

\footnotetext{
*corresponding author; e-mail: papis@ite.waw.pl
}

X-ray photoelectron spectroscopy (XPS), and scanning tunneling microscopy (STM). However, it is not sufficient for a complete characterization.

Understanding the electrical properties is a crucial problem for a number of emerging applications, including molecular electronics and the use of semiconductors as a basis for chemical and biological sensing. Two parameters of particular interest have been the capacitance and the resistance of the organic monolayers. The possibility of using organic monolayers as dielectric has led to interest in characterizing the capacitance of individual monolayers. This is special true for the thiols layers on GaAs, InP, and GaSb semiconductors, which can be applied as the surface passivation treatment $[2,3]$.

On the other hand, the thiols are very sensitive and typing mode, usual for AFM can damage such organic structures.

In this work, the PeakForce Tapping technique was used for study of GaAs and GaSb surfaces treated by hexadecanethiol (HDT) solution. The results of both surface morphology control and electrical properties characterization have been presented.

\section{Experimental}

The surface study was performed on (100) GaSb and (100) GaAs undoped substrates. Prior to the chemical treatment in thiols solution the samples were cleaned in degreasing in hot organic solvents terminated by cold isopropanol rinse and etched sequentially in $30 \mathrm{HCl}+$ $1 \mathrm{HNO}_{3}$ at $T=5^{\circ} \mathrm{C}$ for $t=20 \mathrm{~s}$ and $1 \mathrm{HCl}+6 \mathrm{H}_{2} \mathrm{O}$ at RT for $60 \mathrm{~s}$ Prior to the chemical treatment in HDT$\mathrm{C}_{2} \mathrm{H}_{5} \mathrm{OH}$ the samples were cleaned in degreasing in hot organic solvents terminated by cold isopropanol rinse and etched in $5 \% \mathrm{HCl}$ at RT for $60 \mathrm{~s}$. Then the samples were immersed in $2 \mathrm{mM} \mathrm{HDT}-\mathrm{C}_{2} \mathrm{H}_{5} \mathrm{OH}$ solution and were left there for $12 \mathrm{~h}$ at RT. Then the samples were rinsed with ethanol and dried with dry $\mathrm{N}_{2}$.

The PeakForce TUNA module builds on Bruker exclusive PeakForce Tapping technology to provide the most complete and highest resolution property mapping to date. Available only on Bruker Dimension Icon and MultiMode 8 Atomic Force Microscopes, PeakForce TUNA 
enables, for the first time, quantitative conductivity mapping on fragile samples, such as organic photovoltaics, lithium ion cathodes, and carbon nanotube assemblies, while eliminating the adverse effects caused by sample damage and tip contamination.

PeakForce TUNA offers highest resolution current mapping on the most fragile samples, unmatched repeatability and consistency in nanoelectrical measurements of correlated nanomechanical and nanoelectrical properties.

New solutions make possible measurements of electrical conductivity in the most delicate materials. Recent developments aim to facilitate the work of substances intended for use in fuel cells and lithium batteries.

\subsection{Conception of PeakForce TUNA}

Nanoscale electrical properties are key parameters in many research areas, from solar energy organic photovoltaic compounds and OLED applications to next-generation electrical devices built on nanoscale building blocks. Though unique in its ability to provide high-resolution nanoscale characterization, atomic force microscopy has for the most part been unable to address these parameters quantitatively with conventional techniques.

Conventional AFM conductivity mapping is based on contact mode, which involves lateral forces and leads to sample damage and tip contamination. The result is low spatial resolution and artifacts that mask the desired information. Conductivity measurements at the nanoscale were first enabled with a Contact Mode AFM equipped with a conductive tip and a current-sensing module. Contact-TUNA has been applied in many research and manufacturing laboratories for the analysis of a wide range of materials, for a wide range of applications. For example, traditional Contact Mode TUNA has been used to localize and image electrical defects in semiconductor and data storage devices, to evaluate the uniformity and integrity of thin dielectric films, to characterize piezoelectric and ferroelectric materials, conducting polymers, nanotubes, biomaterials, and others. However, the use of Contact Mode for topographic feedback has proven to be a severe limiting factor. For samples that require low imaging forces in either (or both) the vertical or lateral directions, Contact Mode imaging is not possible, and therefore neither is traditional TUNA imaging.

The Contact Mode limitation applies to the study of many conductive polymers, organics or other soft conducting materials, or loosely bound samples such as nanowires. AFM has achieved tremendous benefits from a variety of oscillating tip modes of operation, most notably TappingMode. During TappingMode imaging, the AFM cantilever is oscillated at its fundamental flexural resonance. This has the advantage of largely eliminating lateral forces that tend to damage the tip and/or sample when imaging in Contact Mode. The vertical interaction force is also substantially reduced due to the high mechanical $Q$ of the cantilever, permitting imaging of soft or delicate samples. When imaging in TappingMode, the AFM tip oscillates relative to the sample by tens of nanometers, and only spends a few percent of that oscillation in contact with the surface. This is advantageous for eliminating tip wear and sample damage, but presents a problem for conductivity measurements.

In order to measure the current signal in such a short time duration $(\mu \mathrm{s})$ with reasonable signal-to-noise ratio, the current amplifier would need a bandwidth in the $\mathrm{MHz}$ range at a high gain $\left(10^{9}-10^{11} \mathrm{~V} / \mathrm{A}\right)$. This is beyond the reach of the current technology. To circumvent this challenge, point-contact current imaging was introduced. In this configuration, TappingMode is used for topographic imaging, and then current-voltage curves are taken at selected points in a Contact Mode fashion.

As in TappingMode, in PeakForce Tapping, the probe and sample are intermittently brought into contact while the tip is scanned across the sample. This eliminates lateral forces during imaging.

Unlike TappingMode, where the feedback loop keeps the average cantilever vibration amplitude constant, in PeakForce Tapping the feedback loop controls the maximum force on the tip (Peak Force) for each individual cycle. Because the force measurement bandwidth of a cantilever is approximately equal to its fundamental resonant frequency, by choosing a modulation frequency significantly lower than the cantilever's resonant frequency, the PeakForce Tapping control algorithm is able to directly respond to the tip-sample force interaction. This direct force control protects the tip and the sample from damage, but more importantly, allows every tip-sample contact to be controlled and recorded for additional mechanical property analysis.

In the current implementation, the modulation frequency is 1 to $2 \mathrm{kHz}$. The foundation of material property mapping with PeakForce QNM is the ability of the system to acquire and analyze the individual force curves from each tip-sample interaction that occurs during the imaging process. The curves are analyzed in real-time to obtain quantitative mechanical properties of the sample, including adhesion, modulus, deformation, and dissipation. These material property maps are treated as conventional AFM channels, and can be displayed and analyzed together with topography. It is important to note that the PeakForce Tapping, oscillation frequency $(1-2 \mathrm{kHz})$ falls nicely between the TappingMode $(>50 \mathrm{kHz})$, and Contact Mode (DC) interaction cycles. In fact, this mid-band operation is the single most important element for TUNA to work in an intermittent contact mode.

In each tapping cycle, the tip is in contact with the sample only for a fraction of the cycle (tens to hundreds of microseconds). The TUNA module must be able to pick up a current signal during this time period with acceptable signal-to-noise ratio. A rule of thumb is that the bandwidth of the TUNA module must be $10 \times$ greater than the tapping frequency at the chosen gain. At TappingMode frequencies this is far beyond the reach of cur- 
rent technology. At PeakForce Tapping speeds, it is an attainable challenge.

The released PeakForce TUNA module is engineered to have a bandwidth around $15 \mathrm{kHz}$ across a range of gains from $10^{7} \mathrm{~V} / \mathrm{A}$ to $10^{10} \mathrm{~V} / \mathrm{A}$, with the noise below $100 \mathrm{fA}$ on cycle-averaged current. The PF-TUNA module has 6 gain settings $\left(10^{7} \mathrm{~V} / \mathrm{A}, 10^{8} \mathrm{~V} / \mathrm{A}, 5 \times 10^{8} \mathrm{~V} / \mathrm{A}, 10^{9} \mathrm{~V} / \mathrm{A}\right.$, $\left.10^{10} \mathrm{~V} / \mathrm{A}, 5 \times 10^{10} \mathrm{~V} / \mathrm{A}\right)$, adjustable through a combination of hardware and software switches. The integration of a wide range of gains on one single module eliminates the need to change modules while searching for the optimal gain to match the conductivity level of a sample, or when different gains are needed to reveal all different conductivity levels present in one single sample. It is noteworthy that the offset at each different gain setting is automatically zeroed out upon each engage, or gain change, to assure measurement accuracy. The PeakForce TUNA module, while designed to work with PeakForce Tapping mode, is compatible with Contact Mode, and provides equal or better noise performance in Contact Mode compared to existing TUNA modules.

Figure 1 illustrates what happens when the periodically modulated PeakForce Tapping probe interacts with the surface. The top line represents the $Z$-position of the cantilever base, as a function of time, as it goes through one period. The middle line represents the force measured by the probe during the approach (blue) and withdraw (red) of the tip to the sample. The bottom line (green) represents the detected current passing through the sample. Since the modulation frequency is about $1 \mathrm{kHz}$, the time from point $A$ to point $E$ is about $1 \mathrm{~ms}$. When the tip is far from the surface (point $A$ ), there is little or no force on the tip. As the tip approaches the surface, the cantilever is pulled down toward the surface by attractive forces (usually van der Waals, electrostatic, or capillary forces) as represented by the negative force (below the horizontal axis). At point $B$, the attractive forces overcome the cantilever stiffness and the tip is pulled to the surface. The tip then stays on the surface and the force increases until the $Z$ position of the modulation reaches its bottom-most position at point $C$. This is where the peak force occurs. The peak force (force at point $C$ ) during the interaction period is kept constant by the system feedback. The probe then starts to withdraw and the force decreases until it reaches a minimum at point $D$. Adhesion is measured by the force at this point. The point where the tip comes off the surface is called the pull-off point. This often coincides with the minimum force. Once the tip has come off the surface, only long range forces affect the tip, so once again, the force is very small or zero when the tip-sample separation is at its maximum (point $E$ ).

From the current-time plot, the PeakForce TUNA algorithm extracts three measurements: (1) peak current, (2) cycle-averaged current, and (3) contact-averaged current. Peak current is the instantaneous current at point $C$, coinciding with peak force. This corresponds to the current measured at a defined force. Peak current

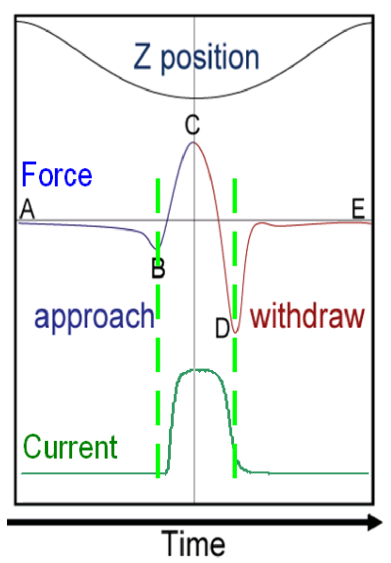

Fig. 1. Plots of $Z$ position, force, and current as a function of time during one PeakForce Tapping cycle, with critical points including $(B)$ jump-to-contact, $(C)$ peak force, $(D)$ adhesion labeled.

may be, but is not necessarily, the maximum current, since the limited rise time (imposed by the bandwidth of the TUNA module or the resistance-capacitance of the sample) may cause a lag in the current response.

Cycle-averaged current is the average current over one full tapping cycle, from point $A$ to point $E$. This includes both the current measured while tip is in contact with the surface, and while it is off the surface.

Contact-averaged current is the average current only when the tip is in contact with the surface, from the snap-in at point $B$ to the pull-off at point $D$. The currenttime plot usually has different characteristics than the force-time plot. It does not always have a peak (as in the force-time plot); current can reach a plateau after a certain force threshold.

There is also an AC current component of the measurement, part of which is capacitive charging, which is removed from the output. The tip (including cantilever) and the sample essentially form a capacitor, and the modulation of $z$-position causes its capacitance to modulate. At a constant DC bias, charging/discharging current at the tapping frequency will occur. Again, this is considered parasitic, and removed as background. The dynamic change of the current (together with deflection) can be captured with NanoScope's "High-Speed Data Capture" function at any time during scanning, and can later be analyzed and correlated (with force for instance).

PeakForce TUNA can be operated in either imaging or spectroscopy mode. In the imaging mode, maps of the electrical current are obtained with topography and mechanical properties. In the spectroscopy mode, one can collect current-voltage $(I-V)$ characteristics.

\subsection{Imaging mode}

In the imaging mode, an electrically conductive probe is scanned over the sample surface in PeakForce Tapping mode as the feedback loop keeps the maximum force (peak force) exerted on the tip at a constant value by adjusting the extension of the $Z$ piezo. This protects the tip 
and sample from damage while allowing the tip sample contact area to be minimized.

During scanning, the user can apply a DC bias between the tip and the sample. The TUNA module, a low-noise, high-bandwidth linear current amplifier, senses the resulting current passing through the sample. This data is presented simultaneously with the topography image and mechanical properties maps (when using PeakForce QNM). The observed current can be used as a measure of the local conductivity, or electrical integrity, of the sample under study. Since the system can acquire up to eight channels simultaneously, it is possible to map the major mechanical properties such as deformation, adhesion, DMT modulus, dissipation, and electrical properties such as cycle-averaged current and peak current together with topography in a single pass.

Offline analysis functions can calculate statistics of the electrical properties of different regions, sections through the data showing the spatial distribution of the properties, and/or correlation between mechanical, topographic and electrical properties.

Here are several tips for using PeakForce TUNA in the imaging mode: (1) use smaller PeakForce setpoints for soft or delicate samples; (2) PeakForce setpoint will affect all three reported current quantities (peak current, cycle-averaged current and contact-averaged current), (3) decreasing PeakForce Tapping amplitude will increase contact time within each tapping cycle, resulting in higher cycle-averaged current and higher contact-averaged current. (4) If simultaneous mechanical properties are desired, a PeakForce set point sufficient to attain a few $\mathrm{nm}$ of deformation is necessary for an accurate DMT Modulus reading.

\section{3. $I-V$ spectroscopy mode}

In addition to the imaging mode, PeakForce TUNA also measures local current-voltage $(I-V)$ spectra using the spectroscopy mode. In order to obtain $I-V$ spectra, the imaging scan is stopped and the tip is held in a fixed location while the sample bias is ramped up or down. In spectroscopy mode, the feedback is switched to Contact Mode, a constant deflection is maintained by the feedback loop while the sample bias is ramped. This assures that tip-sample contact is fixed while $I-V$ curve is taken. The resulting current through the sample is plotted versus the applied bias. The software can either record a single spectrum or average over multiple spectra. The higher bandwidth of the PeakForce TUNA module allows $I-V$ curves to be taken at higher speeds; and it expands the bandwidth of $\mathrm{AC}$ based $\mathrm{d} I / \mathrm{d} V$ measurements, for instance, using the "Generic Lock-in" feature offered with the NanoScopeß $\mathrm{V}$ Controller.

$I-V$ curves can also be taken using the "Point \&Shoot" feature. The "Point \&Shoot" feature offers the option of drawing a line or a box on an image, defining a number of points, and then the AFM tip will automatically move to those locations to capture one or multiple $I-V$ curves at each point. While this is a powerful automation feature, it often can be more useful to "manually" choose a few spots of interest at specific regions on the sample.

\section{Results}

The AFM image (Fig. 2) and the 3D surface roughness parameters (Table) of (100) GaSb show the high quality of surface with rms roughness $=0.265 \mathrm{~nm}$. Dimension Icon system has intrinsic low vertical noise and enables study of such low roughness surface which is not typical for atomic force microscopes able to handle big samples. PeakForce Tapping ensures then ease of use and keeps the tip sharpness due to perfect force control.

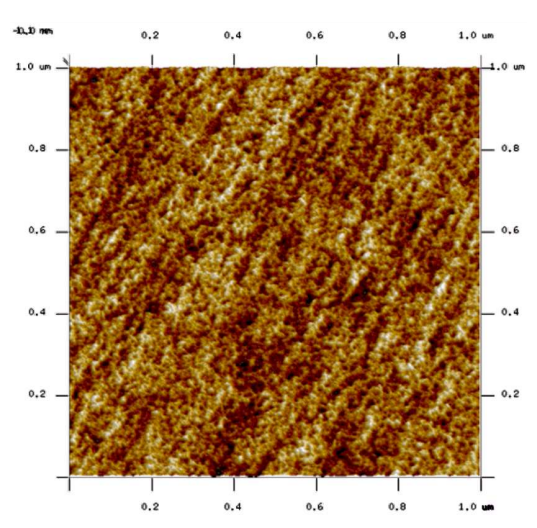

Fig. 2. AFM image of (100) GaSb surface.

TABLE

3D surface roughness parameters.

\begin{tabular}{c|c}
\hline \hline Parameter & Height [nm] \\
\hline$S_{\mathrm{a}}$ & 0.265 \\
$S_{\mathrm{ku}}$ & 3.06 \\
$S_{\mathrm{p}}$ & 1.33 \\
$S_{\mathrm{q}}$ & 0.334 \\
$S_{\mathrm{sk}}$ & -0.114 \\
$S_{\mathrm{v}}$ & -1.50 \\
$S_{\mathrm{z}}$ & 2.83
\end{tabular}

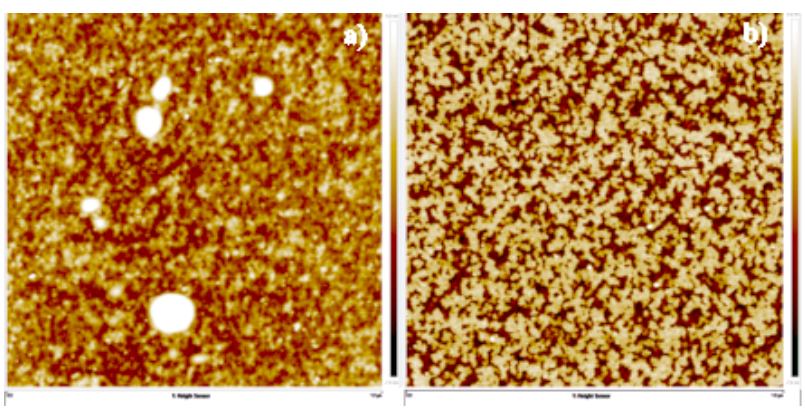

Fig. 3. AFM image of (100) GaAs surface: (a) after HDT $-\mathrm{C}_{2} \mathrm{H}_{5} \mathrm{OH}$ treatment, (b) before $\mathrm{HDT}-\mathrm{C}_{2} \mathrm{H}_{5} \mathrm{OH}$ treatment.

The AFM images of (100) GaAs before and after chemical treatment in $2 \mathrm{mM}$ HDT $-\mathrm{C}_{2} \mathrm{H}_{5} \mathrm{OH}$ have been presented in Fig. 3. The both surfaces are smooth ( $\mathrm{rms}=$ $0.2 \mathrm{~nm}$ ), but on the treated surface the characteristic thiols islands are visible. The images of thiols islands in the micro scale have been presented in another paper in this issue [4]. The adhesion measurements (Fig. 4) indi- 


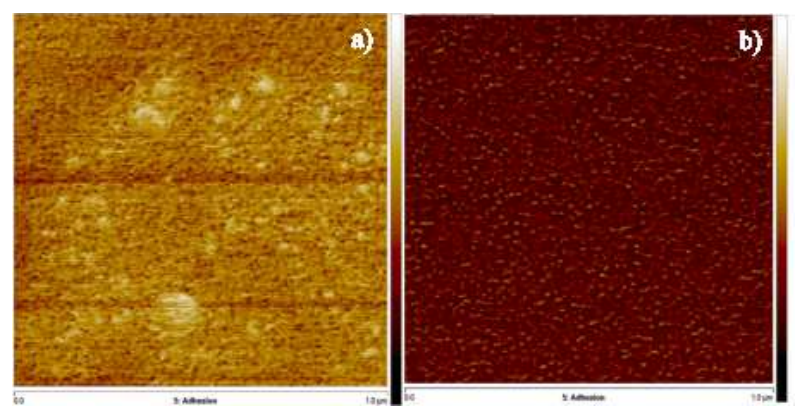

Fig. 4. The image of adhesion of (100) GaAs surface: (a) after $\mathrm{HDT}-\mathrm{C}_{2} \mathrm{H}_{5} \mathrm{OH}$ treatment, (b) before HDT$\mathrm{C}_{2} \mathrm{H}_{5} \mathrm{OH}$ treatment.
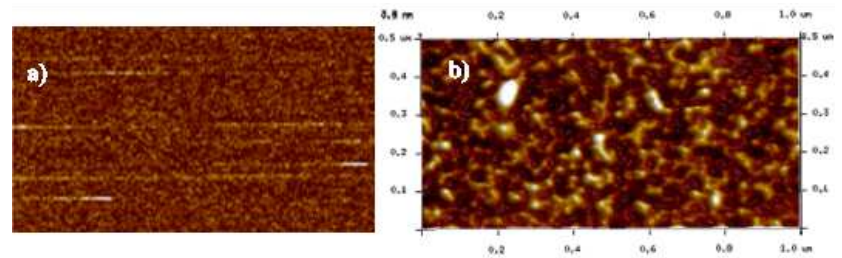

Fig. 5. The images of (100) GaAs surface treated by HDT $-\mathrm{C}_{2} \mathrm{H}_{5} \mathrm{OH}$ : (a) PF TUNA mapping, (b) morphology of surface.

cate the difference of the force: $1.37 \mathrm{nN}$ and $0.66 \mathrm{nN}$ for treated and non-treated GaAs surface, respectively. The measurement is based on the reaction between probe and surface.

Additionally, the contrast of this image is uniform on the mapped surface. It means that GaAs surface is covered by the homogeneous superficial layer. This result is important and consistent with the results of ellipsometric measurements which will be presented in an additional work.

In PF TUNA technique, the mapping image of an electrical conductivity (Fig. 5) is created without any damages of the measured surface and indicates homogeneity of the superficial thiols layer. The force control of Peak Force Tapping ensures the tip to only contact organic layer, which leads to insulator behavior $(I=500 \mathrm{fA})$. Such result would not be possible to obtain with older conductive AFM techniques based on contact mode.

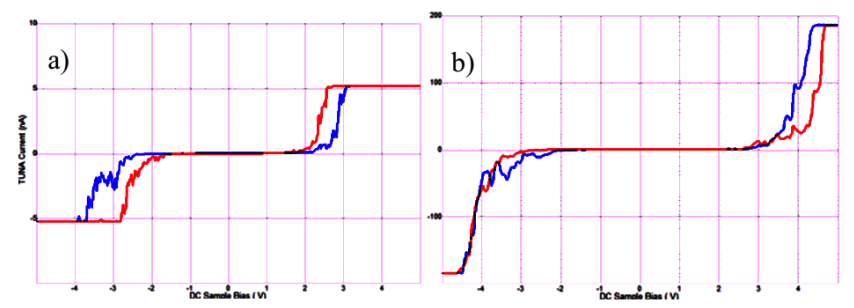

Fig. 6. $I-V$ characteristics for (100) GaAs surface: (a) after HDT- $\mathrm{C}_{2} \mathrm{H}_{5} \mathrm{OH}$ treatment, (b) before HDT$\mathrm{C}_{2} \mathrm{H}_{5} \mathrm{OH}$ treatment.

The $I-V$ characteristics (Fig. 6) show that when the force applied is much higher, the probe is making contact with substrate: as organic (thiols) film prevent oxidation, a conductive behavior is observed and the current measured for GaAs with thiols layer is significantly higher ( $I=5 \mathrm{nA})$ compared with GaAs substrate without thiols $(I=0.2 \mathrm{nA})$.

The images of PF TUNA mapping and surface morphology of untreated GaAs surface have been presented in Fig. 7. There are clearly visible places of higher conductivity confirming that oxide layer on the sample surface is inhomogeneous.

Additionally, an interesting result for treated GaAs surface has been shown in Fig. 8. The electrical field during earlier PF TUNA measurements has modified local chemistry since adhesion is increased, however electrical properties remain unchanged.
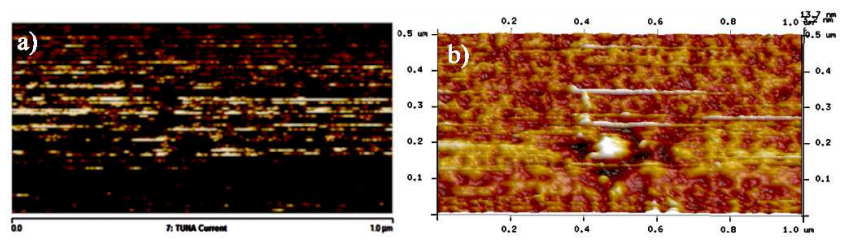

Fig. 7. The images of (100) GaAs surface after HDT treatment: (a) PF TUNA mapping, (b) morphology of surface.

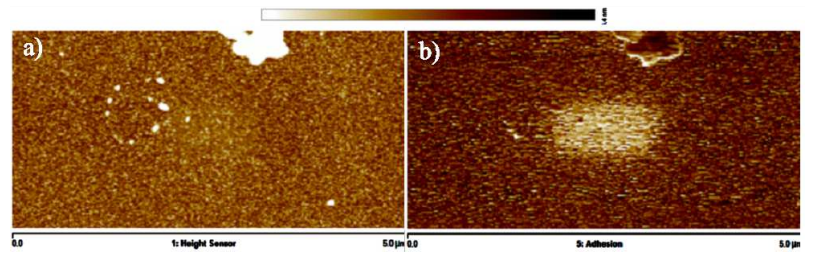

Fig. 8. The images of (100) GaAs surface after HDT$\mathrm{C}_{2} \mathrm{H}_{5} \mathrm{OH}$ treatment: (a) morphology, (b) adhesion.

\section{Summary}

PeakForce Tapping technique was applied for characterization of the semiconductors surface treated by $2 \mathrm{mM}$ HDT $-\mathrm{C}_{2} \mathrm{H}_{5} \mathrm{OH}$. The results of surface morphology, adhesion and $I-V$ characteristics measurements have been presented. This quick, nondestructive method allows to show the high quality of morphology, homogeneous and insulating character of HDT superficial layers on GaAs substrates.

\section{Acknowledgments}

This work was partially supported by the National Centre for Research and Development, Poland under project No. PBS1/B3/2/2012.

\section{References}

[1] R. García, R. Pérez, Surf. Sci. Rep. 4, 197 (2002).

[2] R.V. Ghita, C. Cotirlan, F. Ungureanu, C. Florica, C.C. Negrila, Optoelectron. Adv. Mater., Rapid Commun. 6, 239 (2012).

[3] M. Schvartzman, V. Sidorov, D. Ritter, Y. Paz, J. Vac. Sci. Technol. B 21, 148 (2003).

[4] E. Papis-Polakowska, E. Leonhardt, J. Kaniewski, Acta Phys. Pol. 125, 1052 (2014). 